\title{
Exponentiated Transmuted Generalized Rayleigh Distribution: A New Four Parameter Rayleigh Distribution
}

\author{
Ahmed Z. Afify \\ Department of Statistics, Mathematics and Insurance \\ Benha University, Egypt \\ ahmed.afify@fcom.bu.edu.eg \\ Zohdy M. Nofal \\ Department of Statistics, Mathematics and Insurance \\ Benha University, Egypt \\ dr.zohdynofal@fcom.bu.edu.eg \\ Abd El Hadi N. Ebraheim \\ Institute of Statistical Studies \& Research, \\ Cairo University, Egypt \\ dr.hadi@cu.edu.eg
}

\begin{abstract}
This paper introduces a new four parameter Rayleigh distribution which generalizes the transmuted generalized Rayleigh distribution introduced by Merovci (2014). The new model is referred to as exponentiated transmuted generalized Rayleigh (ETGR) distribution. Various mathematical properties of the new model including ordinary and incomplete moments, quantile function, generating function and Rényi entropy are derived. We proposed the method of maximum likelihood for estimating the model parameters and obtain the observed information matrix. Two real data sets are used to compare the flexibility of the new model versus other models.
\end{abstract}

Keywords: Exponentiated, Generating Function, Rényi Entropy, Moments of residual life, Order Statistics, Maximum Likelihood.

\section{Introduction}

Burr (1942) introduced twelve different forms of cumulative distribution functions for modeling lifetime data. Among those twelve distribution functions, Burr-Type X and BurrType XII received the maximum attention. For more detail about those two distributions seeJohnson et al. (1994). Recently, Surles and Padgett (2001) introduced two-parameter Burr Type X distribution and correctly named as the generalized Rayleigh distribution.

The procedure of expanding a family of distributions for added flexibility or to construct covariate models is a well-known technique in the literature. In many applied sciences such as medicine, engineering and finance, amongst others, modeling and analyzing lifetime data are crucial. Several lifetime distributions have been used to model such kinds of data. The quality of the procedures used in a statistical analysis depends heavily on the assumed probability model or distributions. Because of this, considerable effort has been expended in the development of large classes of standard probability distributions along with relevant statistical methodologies. However, there still remain many important problems where the real data does not follow any of the classical or standard probability models. 
Merovci (2014) introduced transmuted generalized Rayleigh (TGR) distribution. In this article we present a new generalization of the TGR distribution called Exponentiated transmuted generalized Rayleigh (ETGR) distribution.The cumulative distribution function (cdf) of the TGR distribution is given by

$$
G_{T G R}(x, \alpha, \beta, \lambda)=\left\{1-\exp \left[-(\beta x)^{2}\right]\right\}^{\alpha}\left\{1+\lambda-\lambda\left\{1-\exp \left[-(\beta x)^{2}\right]\right\}^{\alpha}\right\}, x>0
$$

where $\alpha, \beta>0,|\lambda| \leq 1$ and $\beta$ is a scale parameter, $\alpha$ is a shape parameter and $\alpha$ the transmuted parameter. The corresponding probability density function (pdf) is given by

$$
\begin{aligned}
& g_{T G R}(x, \alpha, \beta, \lambda)=2 \alpha \beta^{2} x \exp \left[-(\beta x)^{2}\right]\left\{1-\exp \left[-(\beta x)^{2}\right]\right\}^{\alpha-1} \\
& \quad \times\left\{1+\lambda-2 \lambda\left\{1-\exp \left[-(\beta x)^{2}\right]\right\}^{\alpha}\right\} .
\end{aligned}
$$

Recently, the $F^{\alpha}$ distributions (or exponentiated distributions) have been shown to have a wide domain of applicability, in particular in modeling and analysis of lifetime data.

Definition 1: Let $F$ be an absolutely continuous cdf with support on $(a, b)$, where the interval may be unbounded, and let $\alpha$ be a positive real number. The random variable $X$ has an $F^{\alpha}$ distributions if its cdf, denoted by, $G(x)$ is given by

$$
G(x)=F^{\alpha}(x)=[F(x)]^{\alpha}, x>0, \alpha>0 .
$$

which is the $\alpha$ th power of the base line distribution function $F(x)$ and the corresponding pdf of $X$ is given by

$$
g(x)=\alpha f(x)[F(x)]^{\alpha-1} .
$$

The class of $F^{\alpha}$ distributions contains certain well-known distributions for which their cdf's have closed forms (see, e.g. Gupta and Kundu (1999, 2000, 2001, 2007) and Nadarajah (2011)). Shakil and Ahsanullah (2012) introduced some distributional properties of order statistics and record values from $F^{\alpha}$ distributions.

Recently, various generalizations have been introduced based on the above definition. Gupta et al. (1998) first proposed a generalization of the standard exponential distribution, called the exponentiated exponential (EE) distribution. Nadarajah and Kotz (2006) proposed the exponentiated gamma (EГ), exponentiated Fréchet (EF) and exponentiated Gumbel (EGu) distributions. Ebraheim (2014) introduced exponentiated transmuted Weibull distribution. Huang and Oluyede (2014) introduced exponentiated Kumaraswamy-Dagum (EKw-D) distribution.

We aim in this paper to define and study the ETGR distribution. The rest of the paper is organized as follows. In Section 2, we define the new distribution and provide some plots for its pdf. Section 3 provides some statistical properties including quantile function, random number generation, moments, generating functions, incomplete moments, mean 
deviasions and Rényi entropy are derived. In Section 4, the order statistics are discussed. In Section 5, we present the reliability function (rf), hazard rate function (hrf), reversed hazard rate function (rhrf), cumulative hazard rate function (chrf), moments of the residual life and moments of the reversed residual life. The maximum likelihood estimates (MLEs) for the model parameters and the observed information matrix are provided in Section 6. In Section 7, the ETGR distribution is applied to two real data sets to illustrate its usefulness. Finally, some concluding remarks are given in Section 8.

\section{The ETGR Distribution}

The ETGR distribution and its sub-models are presented in this section. The cdf of ETGR (for $X>0$ ) is given by

$$
F(x, \alpha, \beta, \lambda, \delta)=\left\{1-\exp \left[-(\beta x)^{2}\right]\right\}^{\alpha \delta}\left\{1+\lambda-\lambda\left(1-\exp \left[-(\beta x)^{2}\right]\right)^{\alpha}\right\}^{\delta}
$$

Using the series expansion

$$
(1-z)^{-k}=\sum_{j=0}^{\infty} \frac{\Gamma(k+j)}{j ! \Gamma(k)} z^{j}, 0<z<1, k>0 .
$$

The cdf of the ETGR distribution in (3) can be expressed as

$$
F(x, \alpha, \beta, \lambda, \delta)=(1+\lambda)^{\delta} \sum_{K, r=0}^{\infty} v_{j, i}\left(\frac{\lambda}{1+\lambda}\right) \exp \left[-r(\beta x)^{2}\right],
$$

where

$$
v_{j, i}=\frac{(-1)^{k+r} \Gamma(\delta+1) \Gamma(\alpha \delta+\alpha k+1)}{k ! r ! \Gamma(\delta-k+1) \Gamma(\alpha \delta+\alpha k-r+1)}
$$

where $\beta$ is a scale parameter representing the characteristic life, $\alpha$, and $\delta$ are shape parameters representing the different patterns of the ETGR distribution and $\lambda$ the transmuted parameter. The corresponding pdf of (4) is given by

$$
\begin{gathered}
f(x, \alpha, \beta, \lambda, \delta)=2 \alpha \delta \beta^{2} x \exp \left[-(\beta x)^{2}\right]\left\{1-\exp \left[-(\beta x)^{2}\right]\right\}^{\alpha \delta-1} \\
\left\{1+\lambda-2 \lambda\left(1-\exp \left[-(\beta x)^{2}\right]\right)^{\alpha}\right\}\left\{1+\lambda-\lambda\left(1-\exp \left[-(\beta x)^{2}\right]\right)^{\alpha}\right\}^{\delta-1} .
\end{gathered}
$$

Using the series expansion the pdf in (5) can be expressed in the mixture form as

$$
f(x, \alpha, \beta, \lambda, \delta)=2 \alpha \delta(1+\lambda)^{\delta-1} \beta^{2} x\left((1+\lambda) \sum_{j, i=0}^{\infty} b_{j, i}-2 \lambda \sum_{j, i=0}^{\infty} t_{j, i}\right)\left(\frac{\lambda}{1+\lambda}\right)^{j} \exp \left[-(i+1)(\beta x)^{2}\right],
$$

where

$$
b_{j, i}=\frac{(-1)^{j+i} \Gamma(\delta) \Gamma(\alpha \delta+\alpha j)}{j ! i ! \Gamma(\delta-j) \Gamma(\alpha \delta+\alpha j-i)},
$$


and

$$
t_{j, i}=\frac{(-1)^{j+i} \Gamma(\delta) \Gamma(\alpha \delta+\alpha j+\alpha)}{j ! i ! \Gamma(\delta-j) \Gamma(\alpha \delta+\alpha j+\alpha-i)} .
$$

Plots of the pdf for selected parameter values are given in Figure 1.

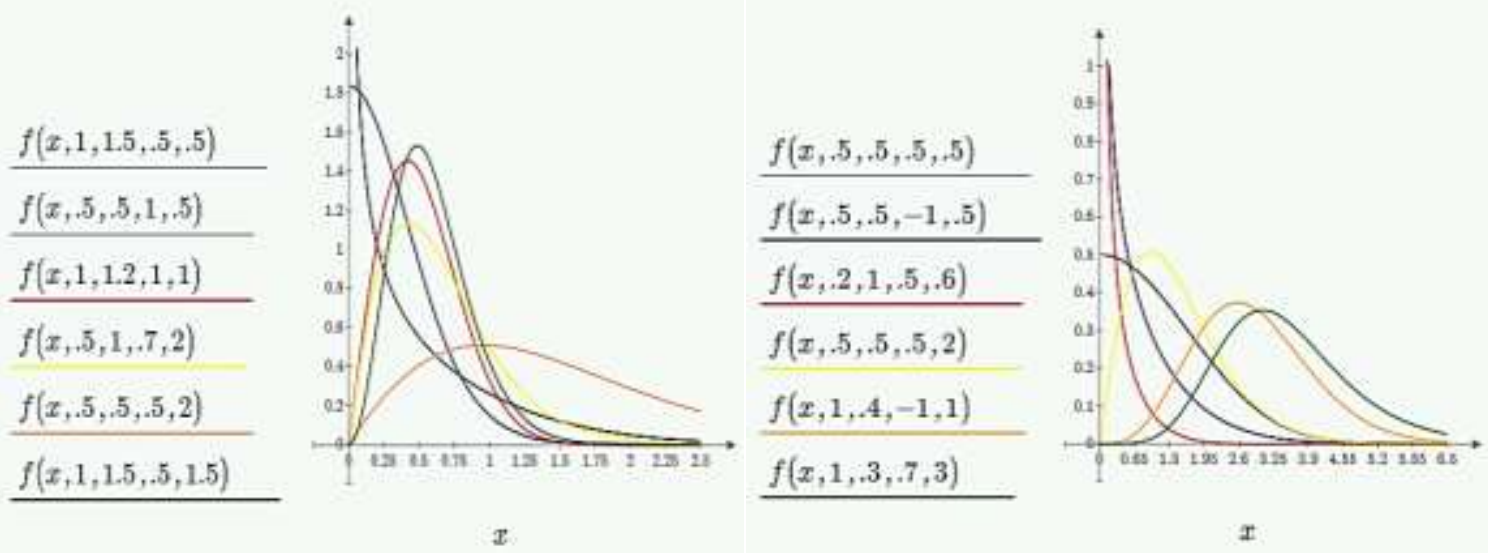

Figure 1: Plots of the density function for some parameter values

The ETGR distribution is very flexible model that approaches to different distributions when its parameters are changed. The subject distribution includes as special cases four well known probability distributions as illustrated in corollary 1.

Corollary 1 If $X$ is a random variable with $\mathrm{pdf}$ in (5), then we have the following seven cases.

1. When $\delta=1$, we get the transmuted generalized Rayleigh distribution, TGR( $\alpha, \beta, \lambda, x)$.

2. When $\delta=1$ and $\lambda=0$, we get the generalized Rayleigh distribution, $\operatorname{GR}(\alpha, \beta, x)$.

3. When $\delta=\alpha=1$, we get the transmuted Rayleigh distribution, $\operatorname{TR}(\beta, \lambda, x)$.

4. When $\delta=\alpha=1$ and $\lambda=0$, we get the Rayleigh distribution, $\mathrm{R}(\beta, x)$.

\section{Statistical Properties}

The statistical properties of the ETGR distribution including quantile and random number generation, moments, moment generating function, incomplete moments, mean deviasions and Rényi entropy are discussed in this section.

\subsection{Quantile and Random Number Generation}


The quantile function (qf), say $x_{q}$, of $X$ is the real solution of the following equation $F\left(x_{q}\right)=q$. Then, we can write

$$
x_{q}=\left(\frac{1}{\beta}\right) \sqrt{-\ln \left(1-\sqrt[\alpha]{\frac{1+\lambda-\sqrt{(1+\lambda)^{2}-4 \lambda q^{1 / \delta}}}{2 \lambda}}\right)}, 0 \leq q \geq 1 .
$$

By putting $q=0.5$ in Equation (7) gives the median of $X$. Simulating the ETGR random variable is straightforward. If $U$ is a uniform variate on the unit interval $(0,1)$, then the random variable $X=X_{q}$ at $q=U$ follows (5).

\subsection{Moments}

The $r$ th moment, denoted by $\mu_{r}$, of $X$ is given by the following theorem.

Theorem 1 If $X$ is a continuous random variable has the $\operatorname{ETGR}(\alpha, \beta, \lambda, \delta, x)$, then the $r$ th non-central moment of $X$, is given by

$$
\mu_{r}^{\prime}=\alpha \delta(1+\lambda)^{\delta-1} \frac{r}{2} \beta^{-r}\left((1+\lambda) \sum_{j, i=0}^{\infty} b_{j . i}-2 \lambda \sum_{j, i=0}^{\infty} t_{j, i}\right)\left(\frac{\lambda}{1+\lambda}\right)^{j}(i+1)^{-(r+2) / 2} \Gamma\left(\frac{r}{2}\right) .
$$

Proof:

By definition

$$
\begin{aligned}
\mu_{r} & =\int_{0}^{\infty} x^{r} f(x, \alpha, \beta, \lambda, \delta) d x \\
& =2 \alpha \delta(1+\lambda)^{\delta-1} \beta^{2}\left((1+\lambda) \sum_{j, i=0}^{\infty} b_{j, i}-2 \lambda \sum_{j, i=0}^{\infty} t_{j, i}\right)\left(\frac{\lambda}{1+\lambda}\right)^{j} I_{x} .
\end{aligned}
$$

where $I_{x}=\int_{0}^{\infty} x^{r+1} \exp \left[-(i+1)(\beta x)^{2}\right] d x$. Using the transformation $t=(\beta x)^{2}$.

Therefore

$$
I_{x}=\int_{0}^{\infty} x^{r+1} \exp \left[-(i+1)(\beta x)^{2}\right] d x=\beta^{-(r+2)}(i+1)^{-(r+2) / 2} \Gamma(1+r / 2) .
$$

By substituting from Equation (10) into Equation (9), we obtain

$$
\mu_{r}=\alpha \delta(1+\lambda)^{\delta-1} \frac{r}{2} \beta^{-r}\left((1+\lambda) \sum_{j, i=0}^{\infty} b_{j . i}-2 \lambda \sum_{j, i=0}^{\infty} t_{j, i}\right)\left(\frac{\lambda}{1+\lambda}\right)^{j}(i+1)^{-(r+2) / 2} \Gamma\left(\frac{r}{2}\right),
$$

Therefore, the first and second moments of the ETGR random variable can be obtained by setting $r=1,2$ respectively, in Equation (8) as follows 


$$
\begin{aligned}
& \mu=\frac{\alpha \delta \pi(1+\lambda)^{\delta-1}}{2 \beta}\left((1+\lambda) \sum_{j, i=0}^{\infty} b_{j . i}-2 \lambda \sum_{j, i=0}^{\infty} t_{j, i}\right)\left(\frac{\lambda}{1+\lambda}\right)^{j}(i+1)^{-3 / 2}, \\
& E\left(X^{2}\right)=\frac{\alpha \delta(1+\lambda)}{\beta^{2}}\left((1+\lambda) \sum_{j, i=0}^{\infty} b_{j . i}-2 \lambda \sum_{j, i=0}^{\infty} t_{j, i}\right)\left(\frac{\lambda}{1+\lambda}\right)^{j}(i+1)^{-2} .
\end{aligned}
$$

Then we can get the variance by the relation $\operatorname{Var}(X)=E\left(X^{2}\right)-E^{2}(X)$.

Based on the above Theorem (1) the coefficient of variation, coefficient of skewness and coefficient of kurtosis of the ETGR $(\alpha, \beta, \lambda, \delta, x)$ distribution can be obtained according to the well-known relations.

Corollary 2 Using the relation between the central moments and non-centeral moments, we can obtain the $n^{\text {th }}$ central moment, denoted by $M_{n}$, of a ETGR random variable as follows

$$
M_{n}=E(X-\mu)^{n}=\sum_{r=0}^{n}\left(\begin{array}{l}
n \\
r
\end{array}\right)(-\mu)^{n-r} E\left(X^{r}\right)
$$

where $E\left(X^{r}\right)$ is the $r$ th non-central moments of $X$. Therefore the $n$th central moments of $X$, is given by

$$
\begin{aligned}
M_{n} & =\alpha \delta(1+\lambda)^{\delta-1} \sum_{r=0}^{n} \frac{\beta^{-r}(-\mu)^{n-r} \Gamma(n+1)}{2 \Gamma(r) \Gamma(n-r+1)}\left\{(1+\lambda) \sum_{j, i=0}^{\infty} b_{j . i}-2 \lambda \sum_{j, i=0}^{\infty} t_{j, i}\right\} \\
& \cdot\left(\frac{\lambda}{1+\lambda}\right)^{j}(i+1)^{-(r+2) / 2} \Gamma\left(\frac{r}{2}\right) .
\end{aligned}
$$

\subsection{Generating Function}

The moment generating function (mgf) of the ETGR distribution is given by the following theorem.

Theorem 2 If $X$ is a continuous random variable has the $\operatorname{ETGR}(\alpha, \beta, \lambda, \delta, x)$, then the moment generating function (mgf) of $X$, denoted by $M_{X}(t)=E\left(e^{t X}\right)$, is given as follows

$$
M_{X}(t)=\alpha \delta(1+\lambda)^{\delta-1} \sum_{r=0}^{\infty} \frac{(i+1)^{-(r+2) / 2}}{\Gamma(r)}\left(\frac{t}{\beta}\right)^{r}\left\{\left(\frac{1+\lambda}{2}\right) \sum_{j, i=0}^{\infty} b_{j, i}-\lambda \sum_{j, i=0}^{\infty} t_{j, i}\right\}\left(\frac{\lambda}{1+\lambda}\right)^{j} \Gamma\left(\frac{r}{2}\right) .
$$

Proof:

By definition 


$$
\begin{gathered}
M_{X}(t)=\int_{0}^{\infty} e^{t x} f(x, \alpha, \beta, \lambda, \delta) d x \\
=\sum_{r=0}^{\infty} \frac{t^{r}}{r !} \int_{0}^{\infty} x^{r} f(x, \alpha, \beta, \lambda, \delta) d x \\
=\sum_{r=0}^{\infty} \frac{t^{r}}{r !} \mu_{r} .
\end{gathered}
$$

By substituting from Equation (8) into Equation (11), we obtain $M_{X}(t)$, which completes the proof. The measure of central tendency, measure of dispersion, coefficient of variation, coefficient of skewness and coefficient of kurtosis of $X$ can be obtained according to the above relation in Theorem 2 .

\subsection{Incomplete Moments}

The main application of the first incomplete moment refers to the Bonferroni and Lorenz curves. These curves are very useful in economics, reliability, demography, insurance and medicine. The answers to many important questions in economics require more than justknowing the mean of the distribution, but its shape as well. This is obvious not only in the study of econometrics but in other areas as well. The sth incomplete moments, denoted by $\varphi_{s}(t)$, of the ETGR r.v. is given by

$$
\varphi_{s}(t)=\int_{0}^{t} x^{s} f(x) d x
$$

Using Equation (6) and the lower incomplete gamma function, we obtain

$$
\begin{aligned}
& \varphi_{s}(t)=\alpha \delta(1+\lambda)^{\delta-1}\left((1+\lambda) \sum_{j, i=0}^{\infty} b_{j . i}-2 \lambda \sum_{j, i=0}^{\infty} t_{j, i}\right) \\
& \times \beta^{-s}(i+1)^{-(s+2) / 2}\left(\frac{\lambda}{1+\lambda}\right)^{j} \Gamma\left(\frac{s}{2}+1,(i+1)(\beta t)^{2}\right) .
\end{aligned}
$$

Another application of the first incomplete moment is related to the mean residual life and the mean waiting time given by $m_{1}(t ; v)=\left(1-\varphi_{1}(t)\right) / R(t ; v)-t \quad$ and $M_{1}(t ; v)=t-\left(\varphi_{1}(t) / F(t ; v)\right)$, respectively.

The amount of scatter in a population is evidently measured to some extent by the totality of deviations from the mean and median. The mean deviations about the mean $\left(\delta_{\mu}(X)=E\left(\left|X-\mu_{1}^{\prime}\right|\right)\right)$ and about the median $\left(\delta_{\mu}(X)=E(|X-M|)\right)$ of $X$ can be, used as measures of spread in a population, expressed by $\delta_{\mu}(X)=\int_{0}^{\infty}\left|X-\mu_{1}^{\prime}\right| f(x) d x=2 \mu_{1}^{\prime} F\left(\mu_{1}^{\prime}\right)-2 \varphi_{1}\left(\mu_{1}^{\prime}\right)$, and

$\delta_{M}(X)=\int_{0}^{\infty}|X-M| f(x) d x=\mu_{1}^{\prime}-2 \varphi_{1}(M)$, respectively, where $\mu_{1}^{\prime}=E(X)$ comes 
from (8), $F\left(\mu_{1}\right)$ is simply calculated from (3), $\varphi_{1}\left(\mu_{1}\right)$ is the first incomplete moments that comes from (12) by setting $s=1$ and $M=$ is the median of $X$.

\subsection{Rényi and q-Entropies}

Entropy refers to the amount of uncertainty associated with a random variable. The Rényi entropy has numerous applications in information theoretic learning, statistics (e.g. classification, distribution identification problems, and statistical inference), computer science (e.g. average case analysis for random databases, pattern recognition, and image matching) and econometrics, see Källberg et al. (2014). The Rényi entropy of a random variable $X$ represents a measure of variation of the uncertainty. The Rényi entropy is defined by

$$
I_{\gamma}(X)=(1-\gamma)^{-1} \log \int_{-\infty}^{\infty} f^{\gamma}(x) d x, \gamma>0 \text { and } \gamma \neq 1
$$

Therefore, using Equation (6), the Rényi entropy of the random variable $X$ is given by

$$
\begin{aligned}
& I_{\delta}(X)=(1-\gamma)^{-1} \log \sum_{j, i, k=0}^{\infty} \zeta_{j i k}\left(2 \alpha \delta \beta^{2}\right)^{\gamma}(1+\lambda)^{\delta \gamma} \\
& \times\left(\frac{2 \lambda}{1+\lambda}\right)^{j}\left(\frac{\lambda}{1+\lambda}\right)^{i} \int_{0}^{\infty} x^{\gamma} \exp \left[-(k+\gamma)(\beta x)^{2}\right] d x,
\end{aligned}
$$

But

$$
\int_{0}^{\infty} x^{\gamma} \exp \left[-(k+\gamma)(\beta x)^{2}\right] d x=\frac{1}{2} \beta^{-(1+\gamma)}(k+\gamma)^{-(1+\gamma) / 2} \Gamma\left(\frac{1+\gamma}{2}\right) .
$$

Then, we can write

$$
I_{\gamma}(X)=(1-\gamma)^{-1} \log \left\{\begin{array}{c}
1 / 2(\alpha \delta)^{\gamma} \beta^{\gamma-1}(1+\lambda)^{\delta \gamma} \Gamma\left(\frac{1+\gamma}{2}\right) \\
\sum_{j, i, k=0}^{\infty} \zeta_{j i k}(k+\gamma)^{-(1+\gamma) / 2}\left(\frac{2 \lambda}{1+\lambda}\right)^{j}\left(\frac{\lambda}{1+\lambda}\right)^{i}
\end{array}\right\},
$$

Where

$$
\zeta_{j i k}=\frac{\left.(-1)^{j+i+k} \Gamma(\gamma+1) \Gamma(\gamma(\delta-1)+1)+1\right) \Gamma(\alpha(j+i+\delta \gamma)-\gamma+1)}{j ! i ! k ! \Gamma(\gamma-j+1) \Gamma(\gamma(\delta-1)-i+1) \Gamma(\alpha(j+i+\delta \gamma)-(\gamma+k)+1)} .
$$

The q-entropy, say $H_{q}(X)$, is defined by

$$
\begin{aligned}
& H_{q}(X)=\frac{1}{q-1} \log \left\{1-\int_{-\infty}^{\infty} f^{q}(x) d x\right\}, q>0 \text { and } q \neq 1 . \\
& H_{q}(X)=\frac{1}{q-1} \log \left\{\begin{array}{c}
1-1 / 2(\alpha \delta)^{q} \beta^{q-1}(1+\lambda)^{\delta q} \Gamma\left(\frac{1+q}{2}\right) \\
\sum_{j, i, k=0}^{\infty} \zeta_{j i k}^{*}(k+q)^{-(1+q) / 2}\left(\frac{2 \lambda}{1+\lambda}\right)^{j}\left(\frac{\lambda}{1+\lambda}\right)^{i}
\end{array}\right\},
\end{aligned}
$$

where 


$$
\zeta_{j i k}^{*}=\frac{\left.(-1)^{j+i+k} \Gamma(q+1) \Gamma(q(\delta-1)+1)+1\right) \Gamma(\alpha(j+i+\delta q)-q+1)}{j ! i ! k ! \Gamma(q-j+1) \Gamma(q(\delta-1)-i+1) \Gamma(\alpha(j+i+\delta q)-(q+k)+1)} .
$$

\section{Order Statistics}

If $X_{1}, X_{2}, \ldots, X_{n}$ is a random sample of size $n$ from a continuous population with cdf $F(x)$ and pdf $f(x)$, and $X_{(1)}, X_{(2)}, \ldots, X_{(n)}$ be the corresponding order statistics. Then the pdf of $X_{(j)}$ is given by

$$
f_{j: n}(x)=\frac{n !}{(j-1) !(n-j) !} f(x)(F(x))^{j-1}(1-F(x))^{n=j}, j=1, \ldots, n .
$$

The joint pdf of $X_{(i: n)}$ and $X_{(j: n)}, 1 \leq i \leq j \leq n$, is given by

$$
\begin{gathered}
f_{i: j: n}(x, y)=\frac{n !}{(i-1) !(j-i-1) !(n-j) !} f(x) f(y) \\
\times(F(x))^{i-1}(F(y)-F(x))^{j-i-1}(1-F(x))^{n-j},
\end{gathered}
$$

for $0 \leq x \leq y \leq \infty$.

The pdf of the $j$ th order statistics for a ETGR distribution is given by

$$
\begin{gathered}
f_{j: n}(x)=\frac{2 \alpha \delta \beta^{2} x n !}{(j-1) !(n-j) !} e^{-(\beta x)^{2}} l_{x}^{\alpha \delta-1}\left(1+\lambda-2 \lambda l_{x}^{\alpha}\right)\left(1+\lambda-\lambda l_{x}^{\alpha}\right)^{\delta-1} \\
.\left(l_{x}^{\alpha \delta}\left(1+\lambda-\lambda l_{x}^{\alpha}\right)^{\delta}\right)^{j-1}\left(1-l_{x}^{\alpha \delta}\left(1+\lambda-\lambda l_{x}^{\alpha}\right)^{\delta}\right)^{n-j}
\end{gathered}
$$

where $l_{x}=\left(1-e^{-(\beta x)^{2}}\right)$.

Therefore, The pdf of the largest order statistics $X_{(n)}$ is

$$
\begin{gathered}
f_{n: n}(x)=2 n \alpha \delta \beta^{2} x e^{-(\beta x)^{2}} l_{x}^{\alpha \delta-1}\left(1+\lambda-2 \lambda l_{x}^{\alpha}\right)\left(1+\lambda-\lambda l_{x}^{\alpha}\right)^{\delta-1} \\
.\left(l_{x}^{\alpha \delta}\left(1+\lambda-\lambda l_{x}^{\alpha}\right)^{\delta}\right)^{n-1}
\end{gathered}
$$

and the pdf of the smallest order statistics $X_{(1)}$ is

$$
\begin{gathered}
f_{1: n}(x)=2 n \alpha \delta \beta^{2} x e^{-(\beta x)^{2}} l_{x}^{\alpha \delta-1}\left(1+\lambda-2 \lambda l_{x}^{\alpha}\right)\left(1+\lambda-\lambda l_{x}^{\alpha}\right)^{\delta-1} \\
.\left(1-l_{x}^{\alpha \delta}\left(1+\lambda-\lambda l_{x}^{\alpha}\right)^{\delta}\right)^{n-1} .
\end{gathered}
$$

The joint pdf of $X_{(i: n)}$ and $X_{(j: n)}, 1 \leq i \leq j \leq n$, for a ETGR distribution is given by 


$$
\begin{aligned}
f_{i: j: n}( & x, y)=\frac{n !\left(2 \alpha \delta \beta^{2}\right)^{2} x y}{(i-1) !(j-i-1) !(n-j) !} e^{-(\beta x)^{2}} e^{-(\beta y)^{2}} l_{x}^{\alpha \delta-1} l_{y}^{\alpha \delta-1} \\
& .\left(1+\lambda-2 \lambda l_{x}^{\alpha}\right)\left(1+\lambda-2 \lambda l_{y}^{\alpha}\right)\left\{\left(1+\lambda-\lambda l_{x}^{\alpha}\right)\left(1+\lambda-\lambda l_{y}^{\alpha}\right)\right\}^{\delta-1} \\
& \cdot\left(l_{x}^{\alpha \delta}\left(1+\lambda-\lambda l_{x}^{\alpha}\right)^{\delta}\right)^{i-1}\left(1-l_{x}^{\alpha \delta}\left(1+\lambda-\lambda l_{x}^{\alpha}\right)^{\delta}\right)^{n-j} \\
& \cdot\left(\left(l_{y}^{\alpha \delta}\left(1+\lambda-\lambda l_{y}^{\alpha}\right)^{\delta}\right)-\left(l_{x}^{\alpha \delta}\left(1+\lambda-\lambda l_{x}^{\alpha}\right)^{\delta}\right)\right)^{j-i-1},
\end{aligned}
$$

where $l_{y}=\left(1-e^{-(\beta y)^{2}}\right)$.

Then the minimum and maximum joint probability density of the ETGR distribution, denoted by $f_{1: n: n}(x, y)$, is

$$
\begin{gathered}
f_{1: n: n}(x, y)=n(n-1)\left(2 \alpha \delta \beta^{2}\right)^{2} x y e^{-(\beta x)^{2}} e^{-(\beta y)^{2}} l_{x}^{\alpha \delta-1} l_{y}^{\alpha \delta-1}\left(1+\lambda-2 \lambda l_{x}^{\alpha}\right) \\
.\left(1+\lambda-2 \lambda l_{y}^{\alpha}\right)\left\{\left(1+\lambda-\lambda l_{x}^{\alpha}\right)\left(1+\lambda-\lambda l_{y}^{\alpha}\right)\right\}^{\delta-1} \\
.\left(\left(l_{y}^{\alpha \delta}\left(1+\lambda-\lambda l_{y}^{\alpha}\right)^{\delta}\right)-\left(l_{x}^{\alpha \delta}\left(1+\lambda-\lambda l_{x}^{\alpha}\right)^{\delta}\right)\right)^{n-2} \cdot
\end{gathered}
$$

Corollary 3 Let $X_{1}, X_{2}, \ldots, X_{n}$ are independently identically distributed ordered random variables from the Exponentiated transmuted generalized Rayleigh distribution having median order $X_{m+1}$ probability density function is given by

$$
\begin{gathered}
f_{m+1: n}(x)=\frac{(2 m+1) !}{m ! m !} f(x)[F(x)]^{m}[1-F(x)]^{m}, 0 \leq x \leq \infty \\
f_{m+1: n}(x)=\frac{(2 m+1) !}{m ! m !} 2 \alpha \delta \beta^{2} x e^{-(\beta x)^{2}} l_{x}^{\alpha \delta-1}\left(1+\lambda-2 \lambda l_{x}^{\alpha}\right)\left(1+\lambda-\lambda l_{x}^{\alpha}\right)^{\delta-1} \\
\cdot\left\{\left(l_{x}^{\alpha \delta}\left(1+\lambda-\lambda l_{x}^{\alpha}\right)^{\delta}\right)\left(1-l_{x}^{\alpha \delta}\left(1+\lambda-\lambda l_{x}^{\alpha}\right)^{\delta}\right)\right\}^{m} .
\end{gathered}
$$

\section{Reliability Analysis}

In this section we introduce the reliability function, the hazard rate function, the cumulative hazard rate function, reversed hazard rate, moments of the residual life and moments of the reversed residual life for the $\operatorname{ETGR}(\alpha, \beta, \lambda, \delta, x)$.

\subsection{The Reliability, Hazard Rate, Reversed Hazard Rate and Cumulative Hazard Rate Functions}

The rf also known as the survival function, which is the probability of an item not failing prior to some time $t$, is defined by $R(x)=1-F(x)$. The rf of the ETGR distribution, say $R(x, \sigma, \lambda, \alpha, a, b)$, can be a useful characterization of life time data analysis. It can be defined as $R(x, \sigma, \lambda, \alpha, a, b)=1-F(x, \sigma, \lambda, \alpha, a, b)$ 


$$
R(x, \sigma, \lambda, \alpha, a, b)=1-\left\{1-\exp \left[-(\beta x)^{2}\right]\right\}^{\alpha \delta}\left\{1+\lambda-\lambda\left(1-\exp \left[-(\beta x)^{2}\right]\right)^{\alpha}\right\}^{\delta}
$$

The other characteristic of interest of a random variable is the hrf. The hrf of the ETGR distribution also known as instantaneous failure rate, say $h(x)$, is an important quantity characterizing life phenomenon. It can be loosely interpreted as the conditional probability of failure, given it has survived to the time $t$. The hrf of the ETGR distribution is defined by $h(x, \sigma, \lambda, \alpha, a, b)=f(x, \sigma, \lambda, \alpha, a, b) / R(x, \sigma, \lambda, \alpha, a, b)$

$h(x, \alpha, \beta, \lambda, \delta)=\frac{\left\{\begin{array}{c}2 \alpha \delta \beta^{2} x e^{-(\beta x)^{2}}\left(1-\exp \left[-(\beta x)^{2}\right]\right)^{\alpha \delta-1}\left\{1+\lambda-2 \lambda\left(1-\exp \left[-(\beta x)^{2}\right]\right)^{\alpha}\right\} \\ \left\{1+\lambda-\lambda\left(1-\exp \left[-(\beta x)^{2}\right]\right)^{\alpha}\right\}^{\delta-1}\end{array}\right\}}{1-\left(1-\exp \left[-(\beta x)^{2}\right]\right)^{\alpha \delta}\left\{1+\lambda-\lambda\left(1-\exp \left[-(\beta x)^{2}\right]\right)^{\alpha}\right\}^{\delta}}$.

It is important to note that the units for $h(x)$ is the probability of failure per unit of time, distance or cycles. These failure rates are defined with different choices of parameters.

Plots of the hazard rate function of ETGR for selected parameter values are provided in Figure 2.

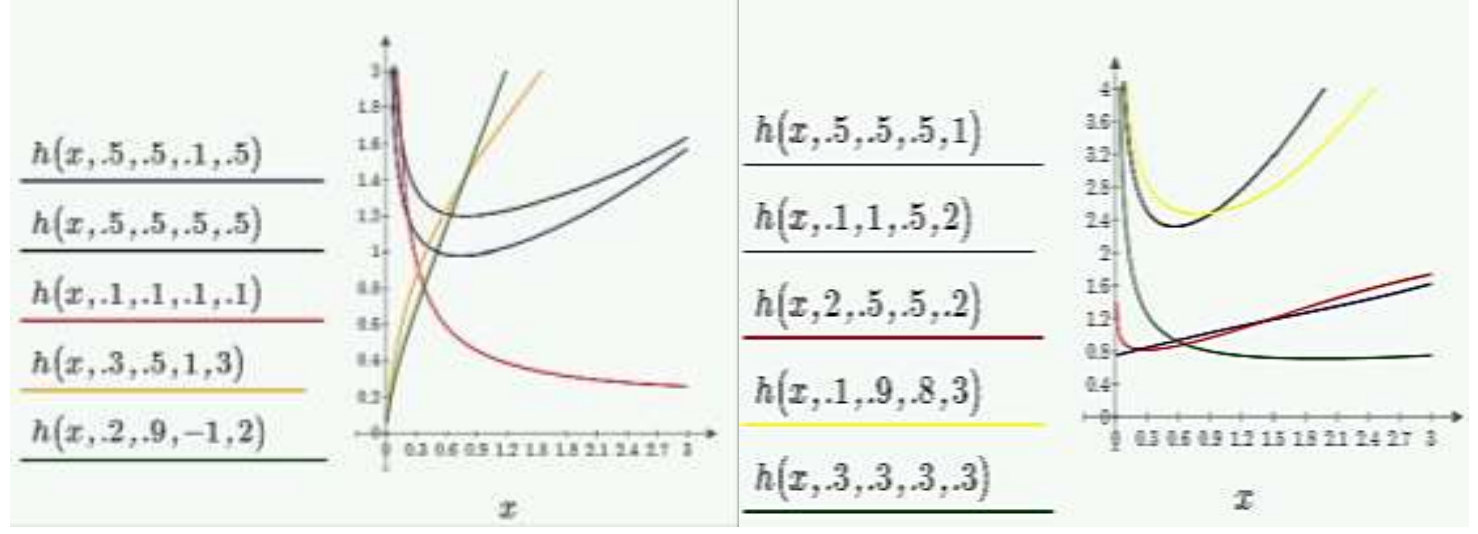

Figure 2: Plots of the hrf for some parameter values

Applications of hrf are quite well known in the statistical literature. Recently the rhrf also becomes quite popular among statisticians, see for example Gupta and Han (2001). Anderson et al. (1993) showed that the rhrf plays the same role in the analysis of leftcensored data as the hazard function plays in the analysis of right-censored data. The rhrf, being the ratio of probability density function and the corresponding distribution function, say $\tau(x)$, is defined by $\tau(x)=f(x) / F(x)$. Therefore, the rhrf is given by 


$$
\tau(x, \alpha, \beta, \lambda, \delta)=\frac{2 \alpha \delta \beta^{2} x \exp \left[-(\beta x)^{2}\right]\left\{1+\lambda-2 \lambda\left(1-\exp \left[-(\beta x)^{2}\right]\right)^{\alpha}\right\}}{\left\{1-\exp \left[-(\beta x)^{2}\right]\right\}\left\{1+\lambda-\lambda\left(1-\exp \left[-(\beta x)^{2}\right]\right)^{\alpha}\right\}}
$$

The chrf of the ETGR distribution, say $H(x, \sigma, \lambda, \alpha, a, b)$, is defined by

$$
\begin{aligned}
& H(x, \sigma, \lambda, \alpha, a, b)=\int_{0}^{x} H(x, \sigma, \lambda, \alpha, a, b) d x=-\ln R(x, \sigma, \lambda, \alpha, a, b) \\
& H(x, \sigma, \lambda, \alpha, a, b)=-\ln \left\{1-\left(1-\exp \left[-(\beta x)^{2}\right]\right)^{\alpha \delta}\left(1+\lambda-\lambda\left(1-\exp \left[-(\beta x)^{2}\right]\right)^{\alpha}\right)^{\delta}\right\} .
\end{aligned}
$$

It is important to note that the units for $H(x, \sigma, \lambda, \alpha, a, b)$ is the cumulative probability of failure or death per unit of time, distance or cycles.

\subsection{Moments of the Residual Life}

Several functions are defined related to the residual life. The failure rate function, mean residual life function and the left censored mean function, also called vitality function. It is well known that these three functions uniquely determine $F(x)$ (see Gupta (1975), Kotz and Shanbhag (1980) and Zoroa et al. (1990)). Other interesting concept is the partial moments, defined by

$$
g_{k}(t)=\int_{t}^{\infty}(x-t)^{k} d F(x), \text { for } k=1,2, \ldots
$$

Some applications of moments in Bayesian point estimation and in management science problems have been mentioned by Winkler et al. (1972). Gupta and Gupta (1983) show that $g_{k}(x)$ uniquely determines $F(x)$.

Definition2 Let $X$ be a random variable (r.v.), usually representing the life length for a certain unit at age $t$ (where this unit can have multiple interpretations), then the r.v. $X_{t}=$ $X-\mid X>t$ represents the remaining lifetime beyond that age.

Moreover, the $k$ th moments of residual life, denoted by $m_{k}(t)=E\left((X-t)^{k} \mid X>t\right), k=1,2,3, \ldots$, uniquely determine $F(x)$ (see Navarro et al. (1998)). The $k$ th moments of the residual life of $X$, say $m_{k}(t)$, is given by

$$
m_{k}(t)=\frac{1}{1-F(t)} \int_{t}^{\infty}(x-t)^{k} d F(x)
$$

Then, we an write 


$$
\begin{aligned}
m_{k}(t) & =\frac{\alpha \delta(1+\lambda)^{\delta-1}}{R(t)} \sum_{r=0}^{k}\left(\begin{array}{l}
k \\
r
\end{array}\right)(-t)^{k-r}\left\{(1+\lambda) \sum_{j, i=0}^{\infty} b_{j . i}-2 \lambda \sum_{j, i=0}^{\infty} t_{j, i}\right\} \\
\times & \beta^{-r}(i+1)^{-(r+2) / 2}\left(\frac{\lambda}{1+\lambda}\right)^{j} \gamma\left(\frac{r+2}{2},(i+1)(\beta t)^{2}\right),
\end{aligned}
$$

where $\gamma(a, b)=\int_{b}^{\infty} y^{a-1} e^{-y} d y$. is the the upper incomplete gamma function.

Another interesting function is the mean residual life function (MRL), defined by $m_{1}(x)=E((X-x) \mid X \geq x)$, and it represents the expected additional life length for a unit which is alive at age $x$. The MRL of the ETGR distribution can be obtained by setting $k=1$ in the above equation. Guess and Proschan (1988) gave an extensive coverage of possible applications of the mean residual life. The MRL has many applications in survival analysis in biomedical sciences, life insurance, maintenance and product quality control, economics and social studies, Demography and product technology (see Lai and Xie (2006)).

\subsection{Moments of the Reversed Residual Life}

The $K$ th moments of the reversed residual life, denoted by $M_{k}(t)=E\left((t-X)^{k} \mid X \leq t\right), k=1,2,3, \ldots$, uniquely determine $F(x)$ (see Navarro et al. (1998)).

The $k$ th moments of the residual life of $X$, say $M_{k}(t)$, is given by

$$
M_{k}(t)=\frac{1}{F(t)} \int_{0}^{t}(t-x)^{k} d F(x) .
$$

Then, The $k$ th moments of the reversed residual life of $X$ is given by

$$
\begin{aligned}
& M_{k}(t)=\frac{\alpha \delta(1+\lambda)^{\delta-1}}{F(t)} \sum_{r=0}^{k}\left(\begin{array}{l}
k \\
r
\end{array}\right)(t)^{k-r}(-1)^{r}\left\{(1+\lambda) \sum_{j, i=0}^{\infty} b_{j . i}-2 \lambda \sum_{j, i=0}^{\infty} t_{j, i}\right\} \\
& \times \beta^{-r}(i+1)^{-(r+2) / 2}\left(\frac{\lambda}{1+\lambda}\right)^{j} \Gamma\left(\frac{r+2}{2},(i+1)(\beta t)^{2}\right) .
\end{aligned}
$$

Body Math The mean waiting time (MWT) also known as mean reversed residual life function, defined by $M_{1}(t)=E((t-X) \mid X \leq t)$, and it represents the waiting time elapsed since the failure of an item on condition that this failure had occurred in $(0, x)$. The MRRL of the ETGR distribution can be obtained by setting $k=1$ in the last equation.

\section{Estimation and Inference}

The maximum likelihood estimators (MLEs) for the parameters of the ETGR distribution is discussed in this section. Consider the random sample $X_{1}, X_{2}, \ldots, X_{n}$ of size $n$ from this distribution with unknown parameter vector $\theta=(\alpha, \beta, \lambda, \delta)^{T}$. Then, the log-likelihood function, say $\ell=\ln \ell(\theta)$, becomes 
$\ell=n(\ln 2+\ln \alpha+\ln \delta+2 \ln \beta)+\sum_{i=1}^{n} \ln x_{i}-\sum_{i=1}^{n}\left(\beta x_{i}\right)^{2}+(\alpha \delta-1) \sum_{i=1}^{n} \ln \{1-$ $\left.\exp \left[-\left(\beta x_{i}\right)^{2}\right]\right\}+\sum_{i=1}^{n} \ln \left\{1+\lambda-2 \lambda\left(1-\exp \left[-\left(\beta x_{i}\right)^{2}\right]\right)^{\alpha}\right\}+(\delta-1) \sum_{i=1}^{n} \ln \{1+\lambda-$ $\left.\lambda\left(1-\exp \left[-\left(\beta x_{i}\right)^{2}\right]\right)^{\alpha}\right\}$

Equation (13) can be maximized either directly by using the R (optim function), SAS (PROC NLMIXED), Ox program (sub-routine MaxBFGS) or by solving the nonlinear likelihood equations obtained by differentiating (13). Therefore, the score vector is given by $\quad U(\theta)=\frac{\partial \ell}{\partial \theta}=\left(\frac{\partial \ell}{\partial \alpha}, \frac{\partial \ell}{\partial \beta}, \frac{\partial \ell}{\partial \lambda}, \frac{\partial \ell}{\partial \delta}\right) . \quad$ Let $S_{i}=\left\{1-\exp \left[-\left(\beta x_{i}\right)^{2}\right]\right\}$,

$$
\begin{gathered}
P_{i}=\left(\frac{2}{\beta}\right)\left(\beta x_{i}\right)^{2} \exp \left[-\left(\beta x_{i}\right)^{2}\right] . \text { Then, } \\
\quad \frac{\partial \ell}{\partial \alpha}=\frac{n}{\alpha}+\delta \sum_{i=1}^{n} \ln S_{i}-2 \lambda \sum_{i=1}^{n} \frac{S_{i}^{\alpha} \ln S_{i}}{1+\lambda-2 \lambda S_{i}^{\alpha}}+\lambda(1-\delta) \sum_{i=1}^{n} \frac{S_{i}^{\alpha} \ln S_{i}}{1+\lambda-\lambda S_{i}^{\alpha}} \\
\quad \frac{\partial \ell}{\partial \beta}=\frac{2}{\beta}\left[n-\sum_{i=1}^{n}\left(\beta x_{i}\right)^{2}\right]+(\alpha \delta-1) \sum_{i=1}^{n} \frac{P_{i}}{S_{i}}-2 \alpha \lambda \sum_{i=1}^{n} \frac{P_{i} S_{i}^{\alpha-1}}{1+\lambda-2 \lambda S_{i}^{\alpha}}+ \\
\alpha \lambda(1-\delta) \sum_{i=1}^{n} \frac{P_{i} S_{i}^{\alpha-1}}{1+\lambda-\lambda S_{i}^{\alpha}} \\
\frac{\partial \ell}{\partial \lambda}=\sum_{i=1}^{n} \frac{1-2 S_{i}^{\alpha}}{1+\lambda-2 \lambda S_{i}^{\alpha}}+(\delta-1) \sum_{i=1}^{n} \frac{1-S_{i}^{\alpha}}{1+\lambda-\lambda S_{i}^{\alpha}}
\end{gathered}
$$

and

$$
\frac{\partial \ell}{\partial \delta}=\frac{n}{\delta}+\alpha \sum_{i=1}^{n} \ln S_{i}+\sum_{i=1}^{n} \ln \left(1+\lambda-\lambda S_{i}^{\alpha}\right) .
$$

The maximum likelihood estimator $\hat{\theta}=(\hat{\alpha}, \hat{\beta}, \hat{\lambda}, \hat{\delta})$ of $\theta=(\alpha, \beta, \lambda, \delta)^{\mathrm{T}}$ is obtained by solving the nonlinear system of equations (14) through (17). These equations cannot be solved analytically and statistical software can be used to solve them numerically by means of iterative techniques such as the Newton-Raphson algorithm. For the four parameters ETGR distribution all the second order derivatives exist.

For interval estimation of the model parameters, we require the $4 \times 4$ observed information matrix, whose elements are derived in appendix A, $J(\theta)=\left\{J_{r s}\right\}($ for $r, s=\alpha, \beta, \lambda, \delta)$. Under standard regularity conditions, the multivariate normal $N_{4}\left(0, J(v)^{-1}\right)$ distribution can be used to construct approximate confidence intervals for the model parameters. Here, $J(\theta)$ is the total observed information matrix evaluated at $\theta$. Therefore, approximate $100(1-\phi) \%$ confidence intervals for $\alpha, \beta, \lambda$ and $\delta$ can be determined as:

$\hat{\alpha} \pm Z_{\frac{\phi}{2}} \sqrt{J_{\alpha \alpha}}, \quad \hat{\beta} \pm Z_{\frac{\phi}{2}} \sqrt{J_{\beta \beta}}, \quad \hat{\lambda} \pm Z_{\frac{\phi}{2}} \sqrt{J_{\lambda \lambda}}$ and $\hat{\delta} \pm Z_{\frac{\phi}{2}} \sqrt{J_{\delta \delta}}$, where $Z_{\frac{\phi}{2}}$ is the upper $\phi$ th percentile of the standard normal distribution.

\section{Applications}


In this section we provide two applications of the ETGR distribution to two real data sets. The first data set, strength data, which were originally reported by Badar and Priest (1982) and it represents the strength measured in GPA for single carbon fibers and impregnated 1000-carbon fiber tows. Single fibers were tested under tension at gauge lengths of $10 \mathrm{~mm}$ (data set 1 ) and $20 \mathrm{~mm}$ (data set 2), with sample sizes $\mathrm{n}=63$ and $\mathrm{m}=74$ respectively. The data are presented below. Several authors analyzed these data sets. Surles and Padgett (1998, 2001), Raqab and Kundu (2005) observed that the generalized Rayleigh distribution works quite well for these strength data. Kundu and Gupta (2006) analyzed these data sets using two-parameter Weibull distribution after subtracting 0.75 from both these data sets. After subtracting 0.75 from all the points of these data sets, Kundu and Gupta (2006) fitted Weibull distribution to both these data sets with equal shape parameters. These two data sets also studied by Rao (2014) to estimation of reliability in multicomponent stressstrength based on generalized. Here I would like to mention that the exact number of (data set 2) is 74 instead of 69, which mentioned in Kundu and Gupta (2009). Here we used these data to compare the proposed ETGR model with TGR, GR and R distributions.

The first data set (gauge lengths of $10 \mathrm{~mm}$ ) from Kundu and Raqab (2009). This data set consists of, 63 observations: 1.901, 2.132, 2.203, 2.228, 2.257, 2.350, 2.361, 2.396, 2.397, $2.445,2.454,2.474,2.518,2.522,2.525,2.532,2.575,2.614,2.616,2.618,2.624,2.659$, $2.675,2.738,2.740,2.856,2.917,2.928,2.937,2.937,2.977,2.996,3.030,3.125,3.139$, $3.145,3.220,3.223,3.235,3.243,3.264,3.272$, 3.294, 3.332, 3.346, 3.377, 3.408, 3.435, $3.493,3.501,3.537,3.554,3.562$, 3.628, 3.852, 3.871, 3.886, 3.971, 4.024, 4.027, 4.225, $4.395,5.020$.

The second data set (gauge lengths of $20 \mathrm{~mm}$ ) is also obtained from Kundu and Raqab (2009). These data set consists of 74 observations: 1.312, 1.314, 1.479, 1.552, 1.700, $1.803,1.861,1.865,1.944,1.958,1.966,1.997,2.006,2.021,2.027,2.055,2.063,2.098$, $2.140,2.179,2.224,2.240,2.253,2.270,2.272,2.274,2.301,2.301,2.359,2.382,2.382$, $2.426,2.434,2.435,2.478,2.490,2.511,2.514,2.535,2.554,2.566,2.570,2.586,2.629$, $2.633,2.642,2.648,2.684,2.697,2.726,2.770,2.773,2.800,2.809,2.818,2.821,2.848$, 2.880, 2.809, 2.818, 2.821, 2.848, 2.880, 2.954, 3.012, 3.067, 3.084, 3.090, 3.096, 3.128, $3.233,3.433,3.585,3.585$.

In order to compare the distributions, we consider some criteria like $-2 \hat{\ell}$ (maximized loglikelihood), AIC (Akaike Information Criterion), CAIC (the consistent Akaike Information Criterion) and $B I C$ (Bayesian information criterion) for the real data set. The model with minimum $A I C$ or $B I C$ or $C A I C$ value is chosen as the best model to fit the data, where

$$
A I C=-2 \hat{\ell}+2 k, B I C=-2 \hat{\ell}+k \log (n)
$$

and

$$
C A I C=-2 \hat{\ell}+2 k n /(n-k-1) .
$$

where $k$ is the number of parameters and $n$ is the sample size.

Table 1 lists the MLEs of the model parameters for ETGR, TGR, GR and R distributions, the corresponding standard errors are given in parentheses. In this table we shall compare 
between the four distributions. The statistics $-2 \hat{\ell}, A I C, B I C$ and $C A I C$ evaluated at the maximum likelihood estimates.

Table 1: MLEs under the considered models and corresponding $-2 \hat{\ell}, A I C, B I C$ and values CAIC (Data set 1)

\begin{tabular}{ccccccccc}
\hline \hline Model & \multicolumn{2}{l}{ Estimates } & \multicolumn{7}{c}{ Measures } \\
\cline { 2 - 8 } & $\hat{\alpha}$ & $\hat{\beta}$ & $\lambda$ & $\delta$ & $-2 \hat{\ell}$ & AIC & BIC & CAIC \\
\hline ETGR & 2.4051 & 0.5347 & 0.5830 & 6.9503 & $\mathbf{1 1 5}$ & $\mathbf{1 2 2 . 9 7 5}$ & 131.5 & $\mathbf{1 2 3 . 6}$ \\
& $(0.88)$ & $(0.051)$ & $(0.207)$ & $(1.488)$ & & & & \\
\hline TGR & 6.2143 & 0.5021 & 0.1207 & & 118.64 & 124.639 & 131.069 & 125.047 \\
& $(1.216)$ & $(0.011)$ & $(0.369)$ & & & & & \\
\hline GR & 6.213 & 0.5145 & & 122.626 & 126.626 & 130.912 & 126.826 \\
& $(0.966)$ & $(0.024)$ & & & & & \\
\hline R & 0.320 & & & 187.044 & 189.044 & 191.187 & 189.11 \\
& & $0.020)$ & & & & \\
\hline \hline
\end{tabular}

Table 2 lists the MLEs of the model parameters for ETGR, TGR, GR and R distributions, the corresponding standard errors are given in parentheses. In this table we shall compare our new model with other sub models.

Table 2: MLEs under the considered models and corresponding $-2 \hat{\ell}, A I C, B I C$ and values CAIC (Data set 2)

\begin{tabular}{|c|c|c|c|c|c|c|c|c|}
\hline \multirow[t]{2}{*}{ Model } & \multicolumn{4}{|c|}{ Estimates } & \multicolumn{4}{|c|}{ Measures } \\
\hline & $\hat{\alpha}$ & $\hat{\beta}$ & $\lambda$ & $\delta$ & $-2 \hat{\ell}$ & $A I C$ & $B I C$ & CAIC \\
\hline \multirow[t]{2}{*}{ ETGR } & 2.1214 & 0.6985 & 0.3201 & 7.790 & 113.4 & 121.352 & 130.6 & 121.9 \\
\hline & $(0.315)$ & $(0.040)$ & $(0.228)$ & (1.727) & & & & \\
\hline \multirow[t]{2}{*}{ TGR } & 5.5052 & 0.6245 & 0.3599 & & 123.61 & 129.61 & 136.5 & 129.95 \\
\hline & $(0.776)$ & $(0.017)$ & $(0.253)$ & & & & & \\
\hline \multirow[t]{2}{*}{ GR } & 7.784 & 0.6445 & & & 135.202 & 139.202 & 143.811 & 139.371 \\
\hline & $(1.625)$ & $(0.024)$ & & & & & & \\
\hline \multirow[t]{2}{*}{$\mathrm{R}$} & & 0.3962 & & & 188.302 & 190.302 & 192.606 & 190.375 \\
\hline & & $(0.023)$ & & & & & & \\
\hline
\end{tabular}

Tables 1 and 2 compare the ETGR model with the TGR, GR, and Rayleigh distributions. We note that the ETGR model gives the lowest values for the $A I C, B I C$ and CAIC statistics among all fitted models. So, we conclude that the ETGR distribution provides a 
superior fit to these data than the other models. These numerical results are obtained using the MATH- CAD PROGRAM.

\section{Conclusions}

In this paper, We propose a new four-parameter distribution, called the exponentiated transmuted generalized Rayleigh (ETGR) distribution, which extends the transmuted generalized Rayleigh (TGR) distribution (Merovci, 2014). An obvious reason for generalizing a standard distribution is the fact that the generalization provides more flexibility to analyze skewed data. We derive some mathematical properties including for the ordinary and incomplete moments, quantile function, generating function, moments of the residual life, moments of the reversed residual life. We furthur study the Rényi and qentropies, using our model as an underlying distribution. The estimation of parameters is approached by the method of maximum likelihood Two numerical examples illustrate that the ETGR distribution can be used quite effectively to provide better fits than its sub models, TGR, GR, and Rayleigh distributions.

\section{References}

1. Badar, M.G. and Priest, A.M. (1982). Statistical aspects of fiber and bundle strength in hybrid composites. In: Hayashi, T., Kawata, K., Umekawa, S. (Eds.), Progress in Science and Engineering Composites. ICCM-IV, Tokyo, pp. 1129-1136.

2. Burr, I. W. (1942). Cumulative frequency functions. The Annals of Mathematical Statistics, 13, 215-232.

3. Ebraheim, A. N. (2014). Exponentiated Transmuted Weibull Distribution: A Generalization of the Weibull Distribution. International Journal of Mathematical, Computational, Physical and Quantum Engineering. Vol. 8, No. 6.

4. Gupta, R.C. (1975). On characterization of distributions by conditional expectations. Comm. Statist. 4 (1), 99-103.

5. Gupta, P.L. and Gupta, R.C. (1983). On the moments of residual life in reliability and some characterization results. Comm. Statist. Theory Methods 12 (4), 449-461.

6. Gupta, R. C., Gupta, P. L. and Gupta, R. D. (1998). Modeling failure time data by Lehmann alternatives. Communications in Statistics - Theory and Methods 27, 887904.

7. Gupta, R.D. and Kundu, D. (1999). Generalized exponential distribution, Austral. \& New Zealand J. Statist., 41, 173-188.

8. Gupta, R.D. and Kundu, D. (2000). Generalized exponential distribution: different method of estimations, J. Statist. Comput. Simul., 00, 122.

9. Gupta, R.D. and Kundu, D. (2000). Exponentiated exponential family: an alternative to gamma and Weibull distributions, Biometrical J., 43, 117130.

10. Gupta, R.D. and Kundu, D. (2007). Generalized exponential distribution: Existing results and some recent developments, J. Statist. Plann. Inference, 137,3537 3547.

11. Guess, F. and Proschan, F. (1988). Mean residual life, theory and applications. In: Krishnaiah, P.R., Rao, C.R. (Eds.), Handbook of Statistics. Vol. 7. Reliability and Quality Control, 215--224. 
12. Johnson, N. L., Kotz, S. and Balakrishnan, N. (1994). Continuous univariate distributions, 1, John Wiley Sons.

13. Källberga, D., Leonenkob, N and Seleznjeva, O. (2014). Statistical Estimation of Quadratic Rényi Entropy for a Stationary M-dependent Sequence. Journal of Nonparametric Statistics. Vol. 26, No. 2, 385--411.

14. Kotz, S. and Shanbhag, D.N. (1980). Some new approaches to probability distributions. Adv. Appl. Prob. 12, 903-921.

15. Kundu, D. and Gupta, R.D. (2005). Estimation of $\mathrm{P}(\mathrm{Y}<\mathrm{X})$ for the generalized exponential distribution. Metrika 61 (3), 291--308.

16. Kundu, D. and Gupta, R.D. (2006). Estimation of $\mathrm{P}(\mathrm{Y}<\mathrm{X})$ for Weibull distribution. IEEE Transactions on Reliability 55 (2), 270--280.

17. Kundu, D. and Raqab, M. Z. (2009). Estimation of $\mathrm{R}=\mathrm{P}(\mathrm{Y}<\mathrm{X})$ for threeparameter Weibull distribution. Statistics and Probability Letters. Vol. 79, 18391846.

18. Lai, C. D. and Xie, M. (2006). Stochastic ageing and dependence for reliability. Springer, New York.

19. Merovci, F. (2014), Transmuted generalized Rayleigh distribution. AJ. Stat. Appl. Pro. 3, No. 1, 9-20.

20. Nadarajah, S. and Kotz, S. (2006). The exponentiated type distributions. Acta Applicandae Mathematicae 92, 97-111.

21. Nadarajah, S. (2011 ). The exponentiated exponential distribution: a survey, AStA Advances in Statistical Analysis, 95, 219251.

22. Navarro, J. Franco, M. and Ruiz, J.M. (1998). Characterization through moments of the residual life and conditional spacing. The Indian Journal of Statistics, Vol. 60, Series A, Pt. 1, 36-48.

23. Rao, G. S. (2014). Estimation of reliability in multicomponent stress-strength based on generalized Rayleigh distribution. Journal of Modern Applied Statistical Methods. Vol. 13, No. 1, 367-379.

24. Raqab, M.Z., Kundu, D., 2005. Comparison of different estimators of P [Y < X ] for a scaled Burr type X distribution. Communications in Statistics. Simulation and Computation 34 (2), 465-483.

25. Shakil, M. and Ahsanullah, M. (2012). Review on order statistics and record values from $F^{\alpha}$ distributions, Pak. J. Stat. Oper. Res. VIII, 101120.

26. Surles, J.G. and Padgett, W.J. (1998). Inference for $\mathrm{P}(\mathrm{Y}<\mathrm{X})$ in the Burr type $\mathrm{X}$ model. Journal of Applied Statistical Sciences 7, 225-238.

27. Surles, J.G. and Padgett, W.J. (2001). Inference for reliability and stress--strength for a scaled Burr-type X distribution. Lifetime Data Analysis 7, 187--200.

28. Winkler, R.L., Roodman, G.M. and Britney, R.R. (1972). The determination of partial moments. Management Science 19 (3), 290-296. 
29. Zoroa, P., Ruiz, J.M. and Marin, J. (1990). A characterization based on conditional expectations. Comm. Statist. Theory Methods 19 (8), 3127-3135. 


\section{Appendix A}

The elements of the observed information matrix are given by

$$
\begin{aligned}
& J_{\alpha \alpha}=\frac{-n}{\alpha^{2}}-\lambda(1+\lambda) \sum_{i=1}^{n} S_{i}^{a}\left(\ln S_{i}\right)^{2}\left(\frac{2}{\left(1+\lambda-2 \lambda S_{i}^{a}\right)^{2}}+\frac{\delta-1}{\left(1+\lambda-\lambda S_{i}^{a}\right)^{2}}\right), \\
& J_{\alpha \beta}=\delta \sum_{i=1}^{n} \frac{P_{i}}{S_{i}}-2 \lambda \sum_{i=1}^{n} \frac{P_{i} S_{i}^{\alpha-1}\left(\alpha(1+\lambda) \ln S_{i}+\left(1+\lambda-2 \lambda S_{i}^{a}\right)\right)}{\left(1+\lambda-2 \lambda S_{i}^{a}\right)^{2}} \\
& +\lambda(1-\delta) \sum_{i=1}^{n} \frac{P_{i} S_{i}^{\alpha-1}\left(\alpha(1+\lambda) \ln S_{i}+\left(1+\lambda-\lambda S_{i}^{a}\right)\right)}{\left(1+\lambda-\lambda S_{i}^{a}\right)^{2}}, \\
& J_{\alpha \lambda}=(1-\delta) \sum_{i=1}^{n} \frac{S_{i}^{\alpha} \ln S_{i}}{\left(1+\lambda-\lambda S_{i}^{a}\right)^{2}}-2 \sum_{i=1}^{n} \frac{S_{i}^{\alpha} \ln S_{i}}{\left(1+\lambda-2 \lambda S_{i}^{a}\right)^{2}}, \\
& J_{\alpha \delta}=\sum_{i=1}^{n} \ln S_{i}-\lambda \sum_{i=1}^{n} \frac{S_{i}^{\alpha} \ln S_{i}}{1+\lambda-\lambda S_{i}^{a}}, \\
& J_{\beta \beta}=\frac{-2 n}{\beta^{2}}-2 \sum_{i=1}^{n} x_{i}^{2}+(\alpha \delta-1) \sum_{i=1}^{n} \frac{P_{i} S_{i}^{\alpha}\left(1 / \beta-2 \beta x_{i}^{2}\right)-P_{i}^{2}}{S_{i}^{2}} \\
& -2 \alpha \lambda \sum_{i=1}^{n} \frac{P_{i}^{2} S_{i}^{\alpha-2}\left((1+\lambda)(\alpha-1)+2 \lambda S_{i}^{a}\right)+P_{i} S_{i}^{\alpha-1}\left(1+\lambda-2 \lambda S_{i}^{a}\right)\left(1 / \beta-2 \beta x_{i}^{2}\right)}{\left(1+\lambda-2 \lambda S_{i}^{a}\right)^{2}} \\
& +\alpha \lambda(1-\delta) \sum_{i=1}^{n} \frac{P_{i}^{2} S_{i}^{\alpha-2}\left((1+\lambda)(\alpha-1)+\lambda S_{i}^{a}\right)+P_{i} S_{i}^{\alpha-1}\left(1+\lambda-\lambda S_{i}^{a}\right)\left(1 / \beta-2 \beta x_{i}^{2}\right)}{\left(1+\lambda-\lambda S_{i}^{a}\right)^{2}}, \\
& J_{\beta \lambda}=\alpha(1-\delta) \sum_{i=1}^{n} \frac{P_{i} S_{i}^{\alpha-1}}{\left(1+\lambda-\lambda S_{i}^{a}\right)^{2}}-2 \alpha \sum_{i=1}^{n} \frac{P_{i} S_{i}^{\alpha-1}}{\left(1+\lambda-2 \lambda S_{i}^{a}\right)^{2}}, \\
& J_{\beta \delta}=\alpha \sum_{i=1}^{n} \frac{P_{i}}{S_{i}}-\alpha \lambda \sum_{i=1}^{n} \frac{P_{i} S_{i}^{\alpha-1}}{1+\lambda-\lambda S_{i}^{a}} \\
& J_{\lambda \lambda}=(1-\delta) \sum_{i=1}^{n} \frac{\left(1-S_{i}^{\alpha}\right)^{2}}{\left(1+\lambda-\lambda S_{i}^{a}\right)^{2}}-\sum_{i=1}^{n} \frac{\left(1-2 S_{i}^{\alpha}\right)^{2}}{\left(1+\lambda-2 \lambda S_{i}^{a}\right)^{2}} \\
& J_{\lambda \delta}=\sum_{i=1}^{n} \frac{1-S_{i}^{\alpha}}{1+\lambda-\lambda S_{i}^{a}}, \text { and } J_{\delta \delta}=\frac{-n}{\delta^{2}} .
\end{aligned}
$$

